\title{
Transatlantica
}

Revue d'études américaines. American Studies Journal

Susan M. Reverby ed. Tuskegee's Truths : Rethinking the Tuskegee Syphilis Study

Chapel Hill : U of North Carolina P, 2000. p 630.

\section{Elizabeth Chamorand}

\section{(2) OpenEdition}

\section{Journals}

Édition électronique

URL : http://journals.openedition.org/transatlantica/363

DOI : $10.4000 /$ transatlantica.363

ISSN : 1765-2766

Éditeur

AFEA

Référence électronique

Elizabeth Chamorand, «Susan M. Reverby ed. Tuskegee's Truths : Rethinking the Tuskegee Syphilis

Study», Transatlantica [En ligne], 1 | 2001, mis en ligne le 23 mars 2006, consulté le 29 avril 2021. URL :

http://journals.openedition.org/transatlantica/363 ; DOI : https://doi.org/10.4000/transatlantica.363

Ce document a été généré automatiquement le 29 avril 2021.

\section{c) (†) $\odot$}

Transatlantica - Revue d'études américaines est mis à disposition selon les termes de la licence Creative Commons Attribution - Pas d'Utilisation Commerciale - Pas de Modification 4.0 International. 


\section{Susan M. Reverby ed. Tuskegee's Truths: Rethinking the Tuskegee Syphilis Study \\ Chapel Hill : U of North Carolina P, 2000. p 630.}

\section{Elizabeth Chamorand}

Pour tenter de comprendre l'étude menée de 1932 à 1972 par le Public Health Service (PHS) sur des ouvriers agricoles noirs de l'Alabama, Susan M. Reverby a rassemblé des documents de première main (dont des extraits de la correspondance et des rapports de médecins du PHS), et des articles de médecins et spécialistes en sciences humaines dont les points de vue différents et parfois contradictoires éclairent l'information fournie par les documents. Tuskegee's Truths rappelle le contexte de dénuement et de ségrégation raciale dans lequel des médecins du PHS, partant d'une hypothèse raciste, suivirent 400 sujets atteints de syphilis et 200 sujets sains servant de témoins. Pour mener cette expérience non thérapeutique, malgré l'existence de traitements reconnus, et pour pratiquer des autopsies, ils trompèrent les patients en leur faisant croire à un traitement prolongé, et s'assurèrent la collaboration du corps médical local, des autorités sanitaires et de l'armée. Treize rapports furent publiés dans des revues scientifiques sans la moindre protestation avant 1965. En 1966, un employé du PHS tenta en vain d'alerter ses supérieurs. En 1969, la radicalisation de la communauté afro-américaine conduisit le PHS à rechercher une collaboration plus grande de médecins afro-américains, puis, lorsque le scandale éclata, à tenter de justifier l'intérêt scientifique de l'étude, de l'excuser en invoquant l'évolution des règles éthiques en matière de recherche, et d'en minimiser les conséquences pour les victimes. Un article replace l'étude de la syphilis dans le contexte de la recherche médicale, de son exploitation des Noirs de l'esclavage à nos jours. Comment en expliquer la longévité malgré les réformes entreprises au PHS ? Absence de communication au sein du PHS, rivalité commerciale pour produire des tests sérologiques, entêtement aveugle de scientifiques à mener l'expérience jusqu'à sa fin ? Comment expliquer le comportement de l'infirmière noire qui chercha à concilier l'inconciliable, ainsi que la collaboration du Tuskegee Institute? Comment expliquer la réticence des autorités à reconnaitre la 
responsabilité du gouvernement après 1972 ? Le rapport officiel de 1973 ne touchait qu'à l'absence de consentement et au refus du PHS d'utiliser la pénicilline. Le procès intenté contre le gouvernement n'aboutit à un réglement qu'en décembre 1974, et il fallut une requête des victimes, outragées par la falsification de l'histoire à la télévision, pour que des excuses officielles soient présentées en 1997.

2 En explorant les causes et les conséquences de cette tragédie emblématique du racisme dans la recherche médicale aux Etats-Unis, Tuskegee's Truths évoque le traumatisme subi par la communauté afro-américaine et relance le débat sur la recherche médicale dans une société et un monde inégalitaires.

INDEX

Thèmes : Recensions

\section{AUTEUR}

ELIZABETH CHAMORAND

Université Grenoble III 